\title{
Angioedema hereditario
}

\author{
Ramón Vives Toledo ${ }^{\mathrm{a}, \mathrm{b}}$, José Vicente Sorlí Guerola ${ }^{\mathrm{a}, \mathrm{c}}$, \\ Lucía Sierra Santos ${ }^{\mathrm{a}, \mathrm{d}}$, Miguel García Ribes ${ }^{\mathrm{a}, \mathrm{b}}$.
}

a Grupo de trabajo SEMFYC de Genética Clínica y Enfermedades Raras. ${ }^{\text {b}}$ Centro de Salud Cotolino II (Castro Urdiales, Cantabria). Servicio Cántabro de Salud. ${ }^{\mathrm{C}}$ Departamento de Medicina Preventiva y Salud Pública, y CIBER obn. Universitat de València, Valencia.

${ }^{\mathrm{D}}$ Centro de Salud de Manzanares el Real, Madrid.

Correspondencia: Miguel García Ribes.

E-mail: gribesm@gmail.com

Recibido el 2 de febrero de 2012.

Aceptado para su publicación el 21 de marzo de 2012.

\section{RESUMEN}

El angioedema hereditario es una enfermedad rara, de origen genético, que se caracteriza por presentar episodios recurrentes de angioedema, sin urticaria y prurito, que fundamentalmente afecta a piel y mucosas de las vías respiratorias altas y del tracto gastrointestinal, y por no disponer de un tratamiento específico.

Exponemos el caso de un paciente varón de 26 años de edad que presenta desde hace más de un año repetidas crisis de urticaria localizada en cuello, cara, extremidades superiores y genitales, con edema facial, que no se asocian a desencadenante alguno. Acude de forma reiterada al servicio de urgencias de Atención Primaria cuando tiene una crisis para la administración de corticoides y antihistamínicos, si bien los últimos episodios parecen refractarios al tratamiento, por lo que ha habido que recurrir a la administración de adrenalina, con resultados discretos, y en una de las ocasiones ha precisado de traslado al servicio de urgencias hospitalario al desarrollar un broncoespasmo que requirió ingreso hospitalario.

Palabras Clave. Angioedema. Enfermedades raras. Atención Primaria.

\section{ABSTRACT}

\section{Hereditary angioedema}

Hereditary angioedema is a rare disease of genetic origin that is characterized by recurrent episodes of angioedema, without urticaria and pruritus, primarily affecting the skin and mucous membranes of the upper respiratory and gastrointestinal tract, and by not having a specific treatment.

We report the case of a male 26-year-old patient, who, for over one year presents repeated episodes of urticaria located in the neck, face, upper extremities and genitals, with facial oedema, and not associated with any trigger whatsoever. He repeatedly comes to primary care emergency service when having a crisis to be treated with corticosteroids and antihistamines, although recent episodes seem refractory to treatment. For this reason it has been necessary to administer adrenaline, with modest results. On one of the occasions, he has required transfer to hospital emergency department because he developed bronchospasm requiring hospitalization.

Key words: Angioedema. Rare Diseases. Primary Health Care.

\section{INTRODUCCIÓN}

Dentro de las enfermedades raras que pueden verse en los Servicios de Urgencias de Atención Primaria, el angioedema hereditario (AEH) se caracterizada por presentar episodios recurrentes de angioedema, sin urticaria ni prurito, que afectan principalmente a la piel de extremidades, cara y genitales, así como a mucosas de las vías respiratorias altas y del tracto gastrointestinal. A menudo, también cursa con dolor e impotencia funcional de las zonas afectadas. Es una entidad a tener en cuenta, ya que sin tratamiento específico (es resistente a corticoides, adrenalina y antihistamínicos) puede alcanzar una mortalidad de hasta un $30 \%$, en la mayor parte de los casos por obstrucción de la vía aérea.

El AEH es una enfermedad rara (su prevalencia estimada es de 1:50.000), de origen genético, que se transmite de forma autosómica dominante, si bien 
el $25 \%$ de los casos resultan de una mutación de novo. Existen tres tipos básicos de $\mathrm{AEH}$, el tipo 1 es el más frecuente ( $85 \%$ ) y se debe a un déficit de proteína C1 inhibidor (prot-C1-inh), mientras que el tipo 2 es debido a un déficit funcional de la protC1-inh con niveles normales o incluso elevados de la misma. La alteración en la prot-C1-inh altera la regulación de la cascada clásica del complemento en la coagulación y la liberación de bradicinina, lo que podría explicar parcialmente la clínica del cuadro ${ }^{1}$. Recientemente ha sido descrito un tipo 3 , dependiente de estrógenos y con niveles de protC1-inh normales ${ }^{2}$.

Se han encontrado asociaciones entre el AEH y enfermedades autoinmunes como la tiroiditis, lupus, síndrome de Sjögren, enfermedad inflamatoria intestinal o algunas formas de glomerulonefritis. $\mathrm{Se}$ ha propuesto que esta asociación puede ser debida a los bajos niveles de C4 que presentan estos pacientes, ya que el C4 es importante en el aclaramiento de inmunocomplejos. Por otra parte, se conocen una serie de factores que pueden desencadenar los ataques. Entre los más comunes se encuentran procedimientos dentales y la intubación. Menos frecuente son la presencia de piercing lingual, el edema genital en mujeres tras mantener relaciones sexuales, montar en bicicleta o a caballo, el estrés, la exposición al frío, ciertos alimentos, la infección por Helicobacter pylori, así como fármacos como los anticonceptivos orales, los IECAS y el tamoxifeno ${ }^{3,4}$.

\section{OBSERVACIONES CLÍNICAS}

Presentamos el caso de un paciente varón de 26 años de edad que acude al servicio de urgencias de atención primaria por un cuadro de angioedema localizado en cara y cuello, de intensidad moderada. A la exploración de la orofaringe, hay un discreto edema glosouvular, si bien no hay signos de disnea y la saturación de oxigeno se mantiene al $98 \%$. Se inicia tratamiento con una ampolla de dexclorfeniramina intramuscular y $40 \mathrm{mg}$. de metilprednisolona endovenosa. Como en los siguientes 30 minutos el cuadro no parece ceder y comienza a presentar sensación de disnea (si bien la saturación se mantiene en niveles normales y la exploración no se modifica), se plantea la administración de adrenalina subcutánea, así como su traslado al servicio de urgencias del hospital de referencia, en ambulancia medicalizada. Durante el traslado, los síntomas comienzan a remitir, si bien queda en observación en el servicio de urgencias hospitalario durante unas horas.
Este paciente refiere que desde hace más de un año ha presentado más de una decena de crisis recurrentes de urticaria localizada en cuello, cara y extremidades superiores, con edema facial, que no se asocian a desencadenante alguno, acudiendo a los servicios de urgencias de atención primaria, donde se las han controlado, si bien en una ocasión tuvo que ser trasladado al servicio de urgencias hospitalario al desarrollar un broncoespasmo que requirió ingreso hospitalario. No se encuentran antecedentes familiares y personales de interés, salvo algún episodio similar autolimitado en la infancia. Sus padres refieren que no recuerdan cómo se trataron en esa ocasión, pero que fue visto por el alergólogo y que "no tenía alergia a nada según las pruebas", por lo que le dieron el alta. En la actualidad, el paciente está en tratamiento con antihistamínicos a dosis altas que no parecen controlar las crisis y está pendiente de ser visto por el servicio de Alergología.

\section{COMENTARIOS}

El AEH es una entidad de debut precoz, el $40 \%$ de los pacientes experimentan el primer episodio antes de los 5 años y un $75 \%$ antes de los 15 años, si bien la repetición de los ataques tras el debut y hasta la segunda década de la vida es poco frecuente, por lo que, junto a su condición de enfermedad rara, tarda en diagnosticarse.

La evolución de las crisis es relativamente rápida (5-7 horas), instaurándose plenamente en las primeras 24 horas y remitiendo en $48-72$ horas. El edema laríngeo puede aparecer como manifestación única o bien asociada a edema de labios, lengua, úvula o paladar blando. Hay descritos unos pocos casos de episodios fulminantes, pero la mayoría de ataques se resuelven antes de una completa obstrucción de la vía aérea. La clínica va desde sensación de nudo o tirantez en la orofaringe en las primeras fases, hasta disnea franca y pérdida de conocimiento en fases avanzadas. La clínica gastrointestinal se presenta en la mayoría de pacientes y pueden ser la principal manifestación en un cuarto de los mismos. Se presentan gran variedad de alteraciones gastrointestinales, reflejo del edema de la mucosa intestinal, tales como cólicos, nauseas, vómitos o diarrea. En menor frecuencia pueden aparecer otras manifestaciones como edema de vejiga y uretra, dolor torácico, cólico renal, derrame pleural o pericarditis ${ }^{5}$.

Los pacientes con AEH pueden ser diagnosticados y manejados por especialistas o por médicos de 
atención primaria en función de las circunstancias individuales. Existen criterios clínicos y de laboratorio para su diagnóstico, los cuales se presentan en la tabla 1. Una historia familiar de angioedema, dolor abdominal y edema lingual recurrentes apoyan el diagnóstico, aunque no es indispensable, porque existen mutaciones de novo. La tabla 2 presenta el diagnóstico diferencial que incluye los procesos que cursen con edema cutáneo y/o laríngeo sin urticaria ${ }^{6}$.
EI AEH puede ser mortal, principalmente por obstrucción de las vías respiratorias, y las opciones de tratamiento son limitadas. Además de prevenir los factores desencadenantes, el tratamiento de los episodios agudos está basado en 3 tipos de fármacos, ninguno de los cuales ha demostrado ser superior a los otros: el concentrado de prot-C1-inh plasmático purificado, antagonistas de los receptores b2-bradicinina (acetato de icatibant vía subcutánea) y los inhibidores de la calicreína (ecallanti-

\section{Criterios diagnósticos del AEH}

(El diagnóstico requiere un criterio clínico y un criterio de laboratorio)

\section{Criterios clínicos:}

- Angioedema subcutáneo no inflamatorio, autolimitado, sin urticaria, recurrente y de duración mayor de 12 horas.

- Dolor abdominal autolimitado sin etiología orgánica, recurrente y de duración mayor de 6 horas.

- Edema laríngeo recurrente.

\section{Criterios analíticos:}

- Niveles de C1 inhibidor por debajo del 50\% de los niveles normales en 2 determinaciones, después del primer año de vida.

- Función del C1 inhibidor menor del 50\% de lo normal en 2 determinaciones, después del primer año de vida.

- Mutación del gen C1 inhibidor (este criterio sólo puede ser usado para el diagnóstico en pacientes < 1 año).

- Niveles bajos de C4 durante las crisis, siendo habitualmente normales en periodos intercrisis.

Tabla 1. Criterios diagnósticos del angioedema hereditario

- Reacciones alérgicas y anafilácticas: generalmente son de rápida progresión, afectación de múltiples órganos, agente etiológico identificable y pueden incluir urticaria y sibilancias (nunca en AEH).

- Angioedema idiopático: es un diagnóstico de exclusión. El estudio del complemento es normal.

- Angioedema inducido por drogas: ACO e IECAS en particular. Estudio de complemento y prot-C1-inh son normales.

- Enfermedades autoinmunes, como LES, polimiositis, dermatomiositis y síndrome de Sjögren pueden presentar edema facial, periorbitario y en manos. La inflamación suele ser más persistente.

- Alteraciones tiroideas: hipo e hipertiroidismo pueden presentar cambios similares al angioedema pero con un curso de semanas a meses y no de manera episódica.

- Síndrome de la vena cava superior: puede presentar edema facial, cuello y extremidades superiores de rápida evolución. No presentan alteraciones en prot-C1-inh y sería previsible un progresivo empeoramiento de la inflamación.

- Tumores de la cabeza: pueden presentar edemas localizados, no presentan alteraciones en prot-C1-inh y suelen evolucionar con un progresivo empeoramiento de la inflamación.

- Otras entidades: como queilitis granulomatosa, síndrome Melkersson-Rosenthal, o triquinosis.

Tabla 2. Diagnóstico diferencial del angioedema hereditario 
de vía subcutánea). En general, se restringen estos tratamientos para alteraciones de cualquier tipo de edema laríngeo, alteraciones moderadas o severas del tracto gastrointestinal o alteraciones cutáneas complejas.

A largo plazo se puede plantear tratamiento como profilaxis mediante antiandrógenos como el danazol, que estimula la producción de prot-C1-inh a nivel hepático; pero está contraindicado en niños y durante el embarazo, por lo que el tratamiento de elección en estos casos son los antifibrinolíticos, como el ácido tranexámico o el ácido aminocaproi- $\operatorname{co}^{5-8}$. El asesoramiento genético puede ser útil para los futuros padres con antecedentes familiares de $\mathrm{AEH}$.

Es importante que desde la Atención Primaria se detecten precozmente casos que reúnan criterios clínicos de esta patología, para confirmar o descartar el diagnostico y poner tratamiento lo antes posible. En la tabla 3 se reúnen una serie de consejos para facilitar a los médicos de familia la detección y manejo de estos pacientes.

- $\quad$ Ante un paciente con episodios recurrentes de angioedema, sin urticaria ni prurito, y/o episodios recurrentes de dolor abdominal conviene valorar niveles de prot-C1-inh y alteraciones del complemento.

- Las crisis de AEH son resistentes al tratamiento con corticoides, adrenalina o antihistamínicos, por lo que, en ausencia de los fármacos específicos, solo está indicado el correcto aislamiento de la vía aérea si llegara a ser necesario.

- Hay que advertir al paciente de que ante cualquier síntoma de ataque laríngeo es fundamental acudir a un servicio médico y, si fuera posible, instruirlos en la autoaplicación de los fármacos específicos en domicilio.

- $\quad$ Es necesario, una vez establecido el diagnóstico, ofertar un estudio familiar; aunque debemos recordar que el $25 \%$ de los casos resultan de mutaciones de novo.

Tabla 3. Abordaje del angioedema hereditario en la consulta de Atención Primaria

\section{BIBLIOGRAFÍA}

1. Atkinson JP, Cicardi M, Zuraw B. Hereditary angioedema: Pathogenesis and diagnosis. UpToDate [base de datos en Internet]. [Updated: Oct 20, 2014] Disponible en: http:// www.uptodate.com/contents/hereditary-angioedemapathogenesis-and-diagnosis?source=search_result\&searc $\mathrm{h}=$ angioedema+hereditario\&selectedTitle $=1 \sim 127$.

2. Miranda AR, Ue AP, Sabbag DV, Furlani Wde J, Souza PK, Rotta O. Hereditary angioedema type III (estrogen-dependent) report of three cases and literature review. An Bras Dermatol. 2013;88(4):578-484.

3. Hemperly SE, Agarwal NS, Xu YY, Zhi YX, Craig TJ. Recent advances in the management of hereditary angioedema. $J$ Am Osteopath Assoc. 2013;113(7):546-555.

4. Johnston DT, Lode H. Progress with management of hereditary angioedema. MMW Fortschr Med. 2013;155 (Supl 1):1-5.

5. Parish LC. Hereditary angioedema: diagnosis and mana- gement-a perspective for the dermatologist. J Am Acad Dermatol. 2011;65(4):843-850.

6. Manual Merck. Delves J. Hereditary and Acquired Angioedema. [Consultado el 01-08-2014]. Disponible en: http:// www.merckmanuals.com/professional/immunology_allergic_disorders/allergic_autoimmune_and_other_hypersensitivity_disorders/hereditary_and_acquired_angioedema. $\mathrm{html}$ ?qt=angioedema\&alt=sh.

7. Bernstein JA, Moellman JJ. Progress in the emergency management of hereditary angioedema: focus on new treatment options in the United States. Postgrad Med. 2012;124(3):91-100.

8. Cicardi M, Craig TJ, Martinez-Saguer I, Hébert J, Longhurst HJ. Review of recent guidelines and consensus statements on hereditary angioedema therapy with focus on self-administration. Int Arch Allergy Immunol. 2013;161 (Supl 1):3-9. 